\title{
ERRATUM: "FAR-IR/SUBMILLIMETER SPECTROSCOPIC COSMOLOGICAL SURVEYS: PREDICTIONS OF INFRARED LINE LUMINOSITY FUNCTIONS FOR $z<4$ GALAXIES” (2012, ApJ, 745, 171)
}

\author{
Luigi Spinoglio ${ }^{1}$, Kalliopi M. Dasyra ${ }^{2}$, Alberto Franceschini ${ }^{3}$, Carlotta Gruppioni $^{4}$, \\ ElisabetTa Valiante ${ }^{5}$, and Kate IsaAK ${ }^{6}$ \\ ${ }^{1}$ Istituto di Fisica dello Spazio Interplanetario, INAF, Via Fosso del Cavaliere 100, I-00133 Roma, Italy; luigi.spinoglio@iaps.inaf.it \\ 2 Observatoire de Paris, LERMA (CNRS:UMR8112), 61 Av. de l'Observatoire, F-75014 Paris, France \\ ${ }^{3}$ Dipartimento di Astronomia, Universitá di Padova, Vicolo dell'Osservatorio 5, I-35122 Padova, Italy \\ ${ }^{4}$ Osservatorio Astronomico di Bologna-INAF, Via Ranzani 1, I-40127, Bologna, Italy \\ ${ }^{5}$ School of Physics and Astronomy, Cardiff University, Queen's Buildings, The Parade, Cardiff CF24 3AA, UK \\ ${ }^{6}$ Scientific Support Office, ESTEC/SRE-S, Keplerlaan 1, NL-2200 AZ Noordwijk, The Netherlands \\ Received 2014 June 5; published 2014 August 6
}

\section{Online-only material: color figures}

In the published version of this article, an error was found in the computation of the total infrared (IR) luminosity. Using the formula from Sanders \& Mirabel (1996), we omitted the multiplication factor 1.8, which resulted in underestimations of the total luminosities. Therefore, we present here the corrected results for the line to continuum luminosity relations, the predicted number of sources that could be detected in spectroscopic cosmological surveys from the SPICA and CCAT telescopes, and the line luminosity functions. Because of the relatively small error, the impact on the conclusions of this article is minor. Moreover, Figure 3 of the original article, showing the IR luminosity function from the Valiante et al. (2009) paper, is incorrect due to a numerical artifact. Therefore, we have revised the figure and included it in this erratum.

For better readability, we report here the whole of Sections 2.3 and 2.4, and the first three paragraphs of Section 3.1.

\subsection{Predicting Continuum Luminosity Functions with the Valiante et al. (2009) Model}

The third model that we used is the backward evolution model of Valiante et al. (2009). It was developed using Spitzer and SCUBA observations, and it has been very successful in predicting Herschel results (Berta et al. 2010; Altieri et al. 2010; Glenn et al. 2010; Oliver et al. 2010). This model allows us to take into account galaxies that are not "pure" starburst or "pure" active galactic nuclei (AGNs), and for which the ratios between IR lines might not be the ones expected assuming "pure" spectral energy distributions. This is because the model considers all infrared galaxies as a single population, assuming that starbursts and AGNs coexist. It then uses an empirical relation to assign to each galaxy the fraction of the IR luminosity that is powered by the AGN for its given luminosity and redshift. This relation was derived using a complete sample of local IRAS galaxies, and extrapolated to high- $z$ using Spitzer and SCUBA observations. Figure 1 shows the IR luminosity function predicted by this model for each $z$ bin.

\subsection{Converting Continuum Luminosity Functions to Line or Feature Luminosity Functions}

The galaxy number counts per redshift and bolometric IR luminosity bin that are predicted by each model need to be converted into line luminosity functions, in order to estimate the number of objects that will be detectable in various lines, and to assess whether several of the open questions presented in Section 1 can be addressed. For this purpose, we derived correlations between line and continuum luminosities, including lines for which such correlations were not previously available in the literature, e.g., that of [Si II] for both AGNs and star-forming galaxies. We examined the polycyclic aromatic hydrocarbon (PAH) feature at $11.25 \mu \mathrm{m}$, the purely rotational $\mathrm{H}_{2}(0-0) S 1$ line at $17.03 \mu \mathrm{m}$, and the [Ne II] $12.8 \mu \mathrm{m}$, [Ne v] $14.3 \mu \mathrm{m},[\mathrm{Ne}$ III] $15.5 \mu \mathrm{m},[\mathrm{S}$ III] $18.7 \mu \mathrm{m},[\mathrm{Ne} \mathrm{v}] 24.3 \mu \mathrm{m}$, [O IV] $26 \mu \mathrm{m}$, [Si II] $34.8 \mu \mathrm{m}$, [O III] $52 \mu \mathrm{m}$, [N III] $57 \mu \mathrm{m}$, [O I] $63 \mu \mathrm{m}$, [O III] $88 \mu \mathrm{m}$, [N II] $121.90 \mu \mathrm{m}$, [O I] $145.52 \mu \mathrm{m}$, and [C II] $157.74 \mu \mathrm{m}$ fine-structure lines. These lines cover a wide parameter space of the critical density versus ionization potential diagram (see Figure 4 of the original article), tracing different astrophysical conditions: from photodissociation regions, to stellar/H II regions, to the AGN, and coronal line regions (Spinoglio \& Malkan 1992). This makes the combination of their ratios useful for the creation of AGNs versus star formation diagnostic diagrams (e.g., Spinoglio \& Malkan 1992; Genzel et al. 1998; Dale et al. 2006; Smith et al. 2007).

For lines at wavelengths shorter than $35 \mu \mathrm{m}$, we used the complete, $12 \mu \mathrm{m}$ selected sample of local Seyfert galaxies (Tommasin et al. 2008, 2010) and the Bernard-Salas et al. (2009) sample of starburst galaxies to calibrate the line luminosities to $L_{\mathrm{IR}}$. These samples have been extensively observed in the mid-IR (MIR) with the Infrared Spectrometer (Houck et al. 2004) on board Spitzer (Werner et al. 2004), and the Spitzer spectra have been reduced and analyzed in a consistent way. For the starburst galaxies, we excluded all objects for which there was evidence for the presence of an AGN from the literature or from the detection of [Ne v] (see Table 1 of Bernard-Salas et al. 2009). For the long-wavelength lines, we used the heterogeneous sample of local galaxies compiled by Brauher et al. (2008) containing all observations collected by the Long Wavelength Spectrometer (LWS; Clegg et al. 1996) on board Infrared Space Observatory (ISO; Kessler et al. 1996). The IR luminosities of the galaxies of our sample have been computed from the IRAS fluxes, using the formula of $L_{\mathrm{IR}}{ }^{7}$ representing the total mid- and far-infrared luminosity (Sanders \& Mirabel 1996). All luminosities are in units of $10^{41} \mathrm{erg} \mathrm{s}^{-1}$.

\footnotetext{
$7 \quad L_{\mathrm{IR}}$ is computed by fitting a single-temperature dust emissivity model $\left(\epsilon \propto v^{-1}\right)$ to the flux in all four IRAS bands, and should be accurate to $\pm 5 \%$ for dust temperatures in the range $25-65 \mathrm{~K}$. We notice that the IR luminosities, as defined above, are model dependent, and therefore could introduce some systematics. However, these do not affect the derived relations, as they are within the given errors.
} 


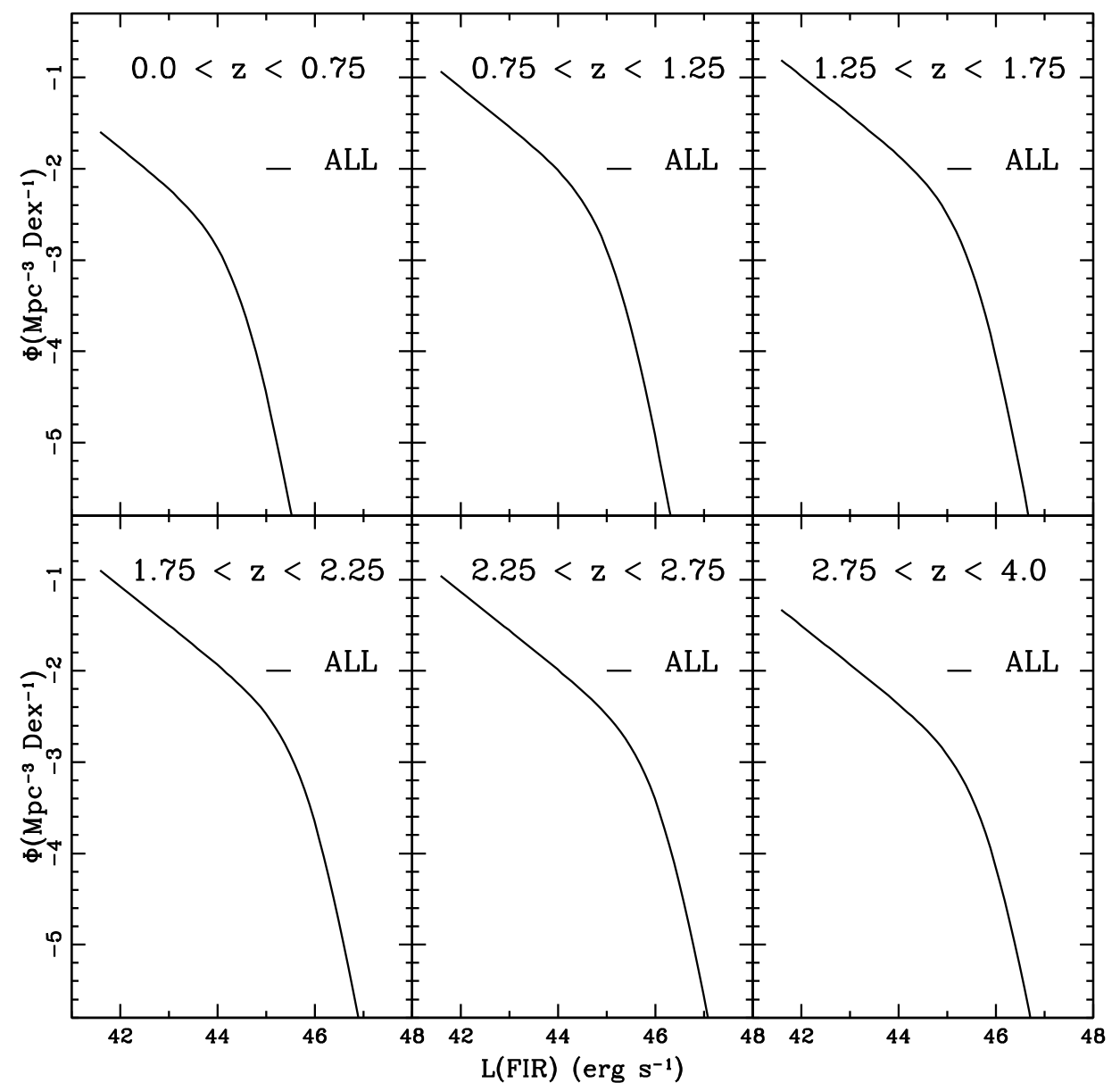

Figure 1. IR luminosity function from Valiante et al. (2009).

Using least-squares fitting, we have obtained the following relations for the Seyfert galaxies ${ }^{8}$ :

$$
\begin{aligned}
& \log \left(L_{\mathrm{PAH} 11.25}\right)=(0.95 \pm 0.07) \log \left(L_{\mathrm{IR}}\right)-(\mathbf{2 . 8 4} \pm \mathbf{0 . 2 3}) \\
& \text { with } R=0.87, n=69, \chi^{2}=7.1 \text {, } \\
& \log \left(L_{[\mathrm{Ne} \text { II }] 12.81}\right)=(0.98 \pm 0.06) \log \left(L_{\mathrm{IR}}\right)-(\mathbf{3 . 5 0} \pm \mathbf{0 . 1 9}) \\
& \text { with } R=0.89, n=87, \chi^{2}=8.8 \text {, } \\
& \log \left(L_{[\mathrm{Nev}] 14.32}\right)=(0.94 \pm 0.08) \log \left(L_{\mathrm{IR}}\right)-(\mathbf{3 . 6 7} \pm \mathbf{0 . 2 7}) \\
& \text { with } R=0.81, n=81, \chi^{2}=14.8 \text {, } \\
& \log \left(L_{\text {[Ne III] } 15.55}\right)=(0.95 \pm 0.07) \log \left(L_{\mathrm{IR}}\right)-(\mathbf{3 . 4 2} \pm \mathbf{0 . 2 5}) \\
& \text { with } R=0.82, n=87, \chi^{2}=15.0 \text {, } \\
& \log \left(L_{\left(\mathrm{H}_{2}\right) 17.03}\right)=(0.97 \pm 0.05) \log \left(L_{\mathrm{IR}}\right)-(\mathbf{4 . 0 4} \pm \mathbf{0 . 1 6}) \\
& \text { with } R=0.92, n=76, \chi^{2}=5.3 \text {, } \\
& \log \left(L_{[\mathrm{S} \mathrm{III}] 18.71}\right)=(0.90 \pm 0.07) \log \left(L_{\mathrm{IR}}\right)-(\mathbf{3 . 5 3} \pm \mathbf{0 . 2 3}) \\
& \text { with } R=0.85, n=70, \chi^{2}=7.7 \text {, } \\
& \log \left(L_{[\mathrm{Nev}] 24.31}\right)=(0.98 \pm 0.08) \log \left(L_{\mathrm{IR}}\right)-(\mathbf{3 . 7 0} \pm \mathbf{0 . 2 6}) \\
& \text { with } R=0.84, n=71, \chi^{2}=10.5 \text {, } \\
& \log \left(L_{[\mathrm{O} \text { Iv }] 25.89}\right)=(0.88 \pm 0.08) \log \left(L_{\mathrm{IR}}\right)-(\mathbf{2 . 8 9} \pm \mathbf{0 . 2 7}) \\
& \text { with } R=0.79, n=83, \chi^{2}=14.9 \text {, }
\end{aligned}
$$

\footnotetext{
8 The bold values indicate the changes with respect to the original article.
} 


$$
\begin{aligned}
& \log \left(L_{[\mathrm{S} I \mathrm{II}] 33.48}\right)=(0.98 \pm 0.05) \log \left(L_{\mathrm{IR}}\right)-(\mathbf{3 . 4 6} \pm \mathbf{0 . 1 8}) \\
& \text { with } R=0.91, n=75, \chi^{2}=5.7 \\
& \log \left(L_{[\mathrm{Si} \text { II] }} 34.82\right)=(1.03 \pm 0.06) \log \left(L_{\mathrm{IR}}\right)-(\mathbf{3 . 4 0} \pm \mathbf{0 . 2 1}) \\
& \text { with } R=0.89, n=72, \chi^{2}=7.3
\end{aligned}
$$

where for each relation the Pearson coefficient $R$, the number of considered objects $n$ and the computed $\chi^{2}$ are given. For the starburst galaxies, the corresponding relations are

$$
\begin{aligned}
& \log \left(L_{\mathrm{PAH} 11.25}\right)=(1.17 \pm 0.11) \log \left(L_{\mathrm{IR}}\right)-(\mathbf{3 . 3 3} \pm \mathbf{0 . 3 5}) \\
& \text { with } R=0.95, n=14, \chi^{2}=1.4 \text {, } \\
& \left.\log \left(L_{[\mathrm{Ne}} \mathrm{II}\right] 12.81\right)=(1.17 \pm 0.14) \log \left(L_{\mathrm{IR}}\right)-(\mathbf{3 . 9 4} \pm \mathbf{0 . 4 3}) \\
& \text { with } R=0.93, n=14, \chi^{2}=2.1 \text {, } \\
& \log \left(L_{[\mathrm{Ne} \mathrm{III]}] 15.55}\right)=(1.33 \pm 0.18) \log \left(L_{\mathrm{IR}}\right)-(\mathbf{5 . 1 9} \pm \mathbf{0 . 5 6}) \\
& \text { with } R=0.90, n=15, \chi^{2}=3.9 \text {, } \\
& \log \left(L_{\left(\mathrm{H}_{2}\right) 17.03}\right)=(1.28 \pm 0.14) \log \left(L_{\mathrm{IR}}\right)-(\mathbf{5 . 4 2} \pm \mathbf{0 . 4 6}) \\
& \text { with } R=0.93, n=15, \chi^{2}=2.6 \text {, } \\
& \log \left(L_{[\mathrm{S} \text { III] }} 18.71\right)=(1.09 \pm 0.15) \log \left(L_{\mathrm{IR}}\right)-(\mathbf{4 . 0 7} \pm \mathbf{0 . 4 9}) \\
& \text { with } R=0.89, n=15, \chi^{2}=3.0 \text {, } \\
& \log \left(L_{[\mathrm{O} \text { Iv }] 25.89}\right)=(1.24 \pm 0.24) \log \left(L_{\mathrm{IR}}\right)-(\mathbf{5 . 4 5} \pm \mathbf{0 . 8 0}) \\
& \text { with } R=0.85, n=12, \chi^{2}=1.6 \\
& \log \left(L_{[\mathrm{S} \text { III] }} 33.48\right)=(1.09 \pm 0.10) \log \left(L_{\mathrm{IR}}\right)-(\mathbf{3 . 6 3} \pm \mathbf{0 . 3 2}) \\
& \text { with } R=0.95, n=15, \chi^{2}=1.2 \text {, } \\
& \log \left(L_{[\mathrm{Si} \text { II] }}{ }_{44.82}\right)=(1.11 \pm 0.09) \log \left(L_{\mathrm{IR}}\right)-(\mathbf{3 . 5 5} \pm \mathbf{0 . 2 7}) \\
& \text { with } R=0.96, n=15, \chi^{2}=0.92 \text {. }
\end{aligned}
$$

The best-fit solution is shown in Figure 2 for each of the populations. Considering the sum of the populations, we derived the following generic relations:

$$
\begin{aligned}
& \log \left(L_{\mathrm{PAH} 11.25}\right)=(0.98 \pm 0.06) \log \left(L_{\mathrm{IR}}\right)-(\mathbf{2 . 9 4} \pm \mathbf{0 . 2 0}) \\
& \text { with } R=0.88, n=83 \chi^{2}=9.4 \text {, } \\
& \left.\log \left(L_{[\mathrm{Ne}} \mathrm{I}\right] 12.81\right)=(0.99 \pm 0.06) \log \left(L_{\mathrm{IR}}\right)-(\mathbf{3 . 5 2} \pm \mathbf{0 . 2 2}) \\
& \text { with } R=0.84, n=101, \chi^{2}=17.8 \text {, } \\
& \log \left(L_{[\mathrm{Ne} \mathrm{III]}] 15.55}\right)=(1.10 \pm 0.07) \log \left(L_{\mathrm{IR}}\right)-(\mathbf{4 . 0 0} \pm \mathbf{0 . 2 5}) \\
& \text { with } R=0.83, n=102, \chi^{2}=24.2 \text {, } \\
& \log \left(L_{\left(\mathrm{H}_{2}\right) 17.03}\right)=(1.07 \pm 0.05) \log \left(L_{\mathrm{IR}}\right)-(\mathbf{4 . 4 7} \pm \mathbf{0 . 1 8}) \\
& \text { with } R=0.91, n=91, \chi^{2}=10.6 \text {, } \\
& \log \left(L_{[\mathrm{S} \text { III }] 18.71}\right)=(0.97 \pm 0.06) \log \left(L_{\mathrm{IR}}\right)-(\mathbf{3 . 7 2} \pm \mathbf{0 . 2 2}) \\
& \text { with } R=0.88, n=70, \chi^{2}=7.9 \text {, } \\
& \log \left(L_{[\mathrm{O} \text { Iv }] 25.89}\right)=(0.95 \pm 0.11) \log \left(L_{\mathrm{IR}}\right)-(\mathbf{3 . 2 8} \pm \mathbf{0 . 3 7}) \\
& \text { with } R=0.68, n=95, \chi^{2}=36.6 \text {, } \\
& \log \left(L_{[\mathrm{S} \mathrm{III}] 33.48}\right)=(0.99 \pm 0.05) \log \left(L_{\mathrm{IR}}\right)-(\mathbf{3 . 4 6} \pm \mathbf{0 . 1 6}) \\
& \text { with } R=0.92, n=90, \chi^{2}=7.4 \text {, }
\end{aligned}
$$



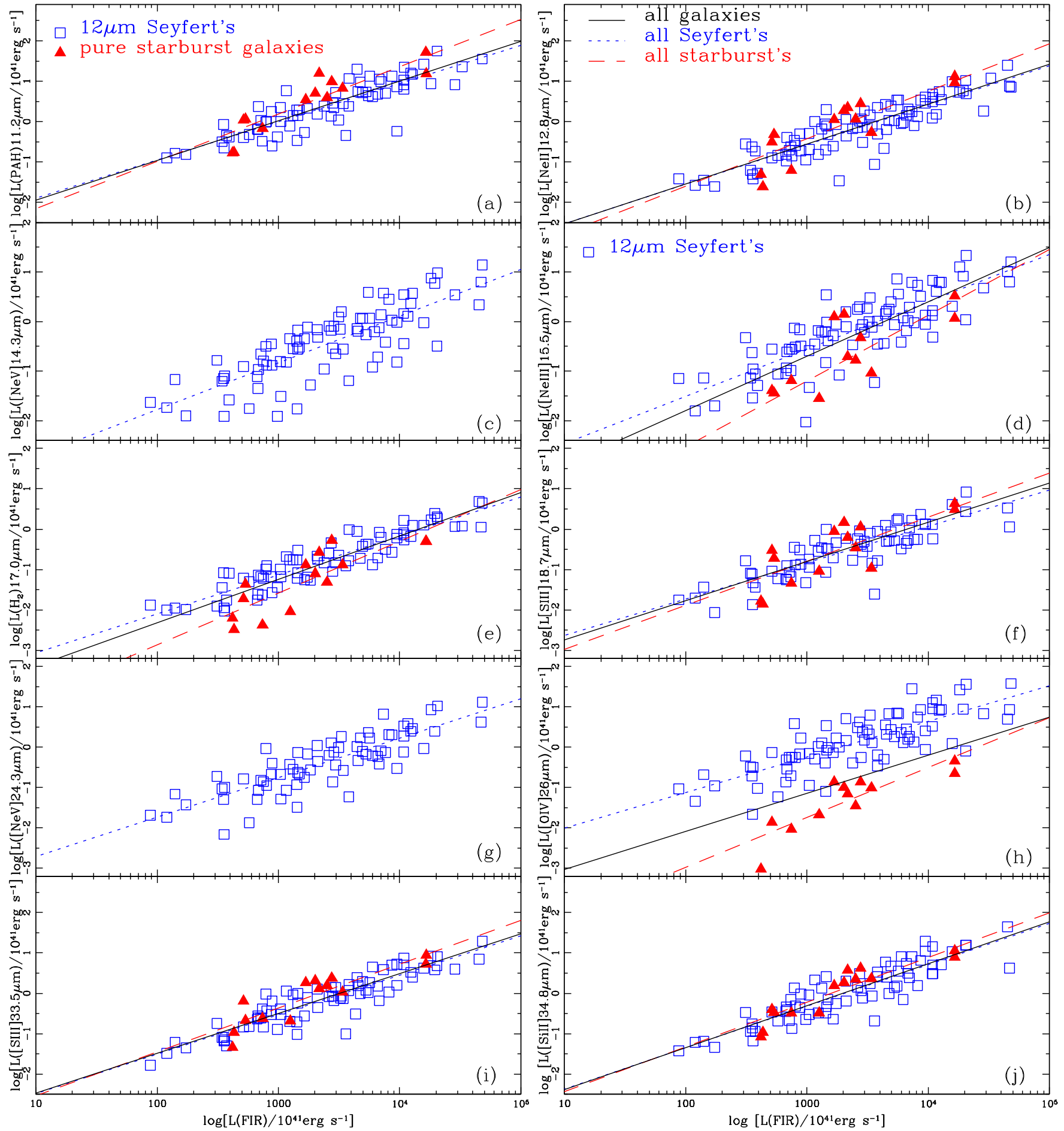

Figure 2. Correlations between the various feature and line luminosities and the far-IR luminosity for the Seyfert galaxies of the complete $12 \mu \mathrm{m}$ galaxy sample (Tommasin et al. 2008, 2010), and for the pure starburst galaxies of the sample of Bernard-Salas et al. (2009). The dotted, broken, and solid lines represent the least-squares fit of the data of the Seyfert, the pure starburst galaxies, and all galaxies populations together, respectively. Panels (c) and (g) have only the Seyfert galaxies, because the $[\mathrm{Ne} v]$ lines are not detected in starburst galaxies.

(A color version of this figure is available in the online journal.)

$$
\begin{aligned}
& \log \left(L_{[\mathrm{Si} \mathrm{II}] 34.82}\right)=(1.04 \pm 0.05) \log \left(L_{\mathrm{IR}}\right)-(\mathbf{3 . 4 2} \pm \mathbf{0 . 1 7}) \\
& \text { with } R=0.91, n=87, \chi^{2}=8.4
\end{aligned}
$$

For the far-IR lines (Figure 3), we obtain

$$
\begin{aligned}
& \left.\log \left(L_{[\mathrm{O}} \mathrm{II}\right] 51.81\right)=(0.88 \pm 0.10) \log \left(L_{\mathrm{IR}}\right)-(\mathbf{2 . 7 6} \pm \mathbf{0 . 3 3}) \\
& \text { with } R=0.91, n=16, \chi^{2}=2.6
\end{aligned}
$$



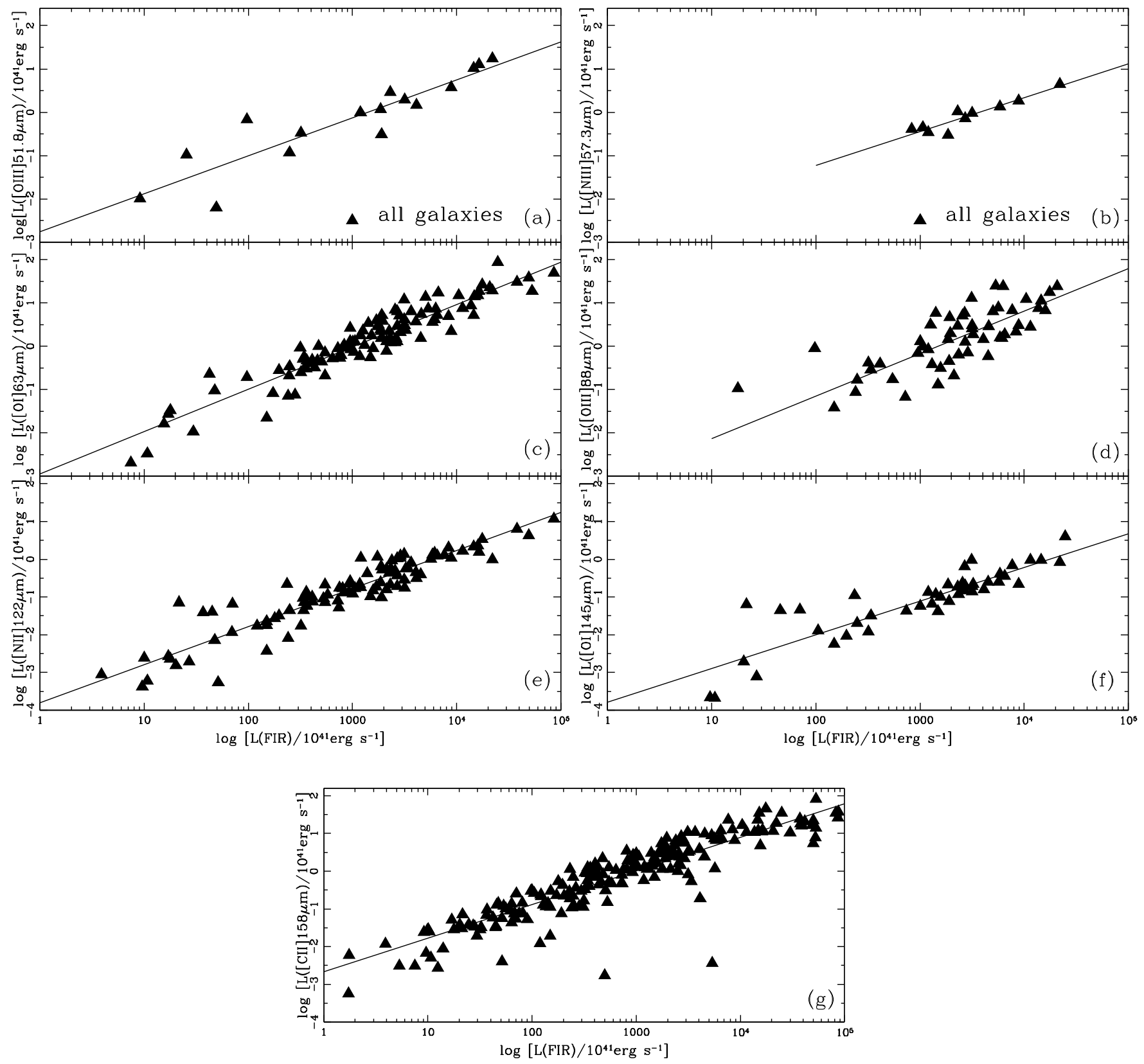

Figure 3. Correlations between the [O III] $52 \mu \mathrm{m}$, [N III] $57 \mu \mathrm{m}$, [O I] $63 \mu \mathrm{m},[\mathrm{O}$ III] $88 \mu \mathrm{m}$, [N II] $122 \mu \mathrm{m},[\mathrm{O}$ I] $145 \mu \mathrm{m}$, and [C II] $158 \mu \mathrm{m}$ luminosity and the far-IR luminosity for the galaxies observed with the ISO-LWS spectrometer (Brauher et al. 2008).

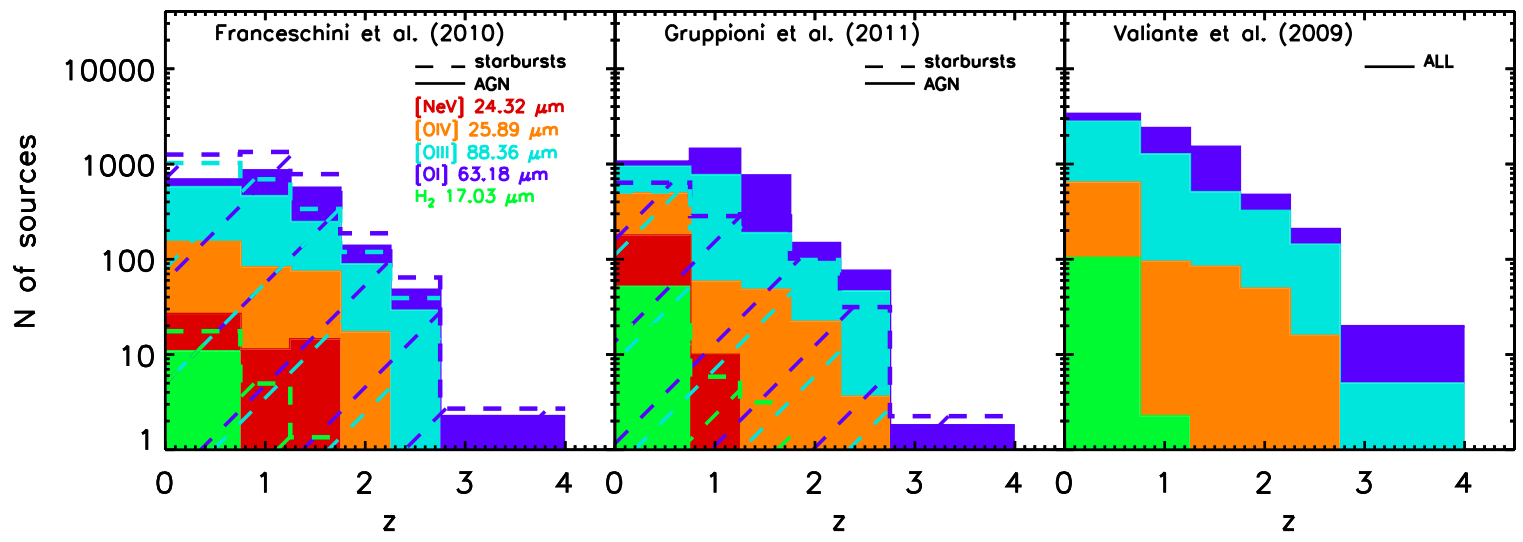

Figure 4. Number of objects detected per spectral line (and per object type, when applicable) in an hour-long/FoV integration covering 0.5 deg $^{2}$ survey with SPICA SAFARI.

(A color version of this figure is available in the online journal.) 
Table 1

Number of AGNs Detectable in a SAFARI Survey of $0.5 \mathrm{deg}^{2}\left(450\right.$ Pointings with a FoV of $\left.2^{\prime} \times 2^{\prime}\right)$ in IR Lines as a Function of Redshift at $5 \sigma(3 \sigma)$ in $1 \mathrm{hr}$ Integration per FoV, Following Franceschini et al. (2010)

\begin{tabular}{|c|c|c|c|c|c|c|c|}
\hline Line/Redshift & $0<z<0.75$ & $0.75<z<1.25$ & $1.25<z<1.75$ & $1.75<z<2.25$ & $2.25<z<2.75$ & $2.75<z<4$ & All Redshifts \\
\hline PAH(11.25 $\mu \mathrm{m})$ & 463. (687.) $\dagger$ & 333. (633.) $\dagger$ & 174. (333.) $\dagger$ & $6.75(54.0)$ & $0.90(5.85)$ & $\cdots(\cdots)$ & $7.65(60.0) \ddagger$ \\
\hline [Ne II] $12.81 \mu \mathrm{m}$ & $69.8(152.) \dagger$ & $35.1(122.) \dagger$ & $0.90(14.4)$ & $\cdots(0.45)$ & $\cdots(\cdots)$ & $\cdots(\cdots)$ & $0.90(14.9) \ddagger$ \\
\hline$[\mathrm{Ne} \mathrm{v}] 14.32 \mu \mathrm{m}$ & $17.6(43.2) \dagger$ & $6.75(20.2) \dagger$ & $\ldots(0.45)$ & $\cdots(\cdots)$ & $\cdots(\cdots)$ & $\cdots(\cdots)$ & $\cdots(0.45) \ddagger$ \\
\hline [Ne III] $15.55 \mu \mathrm{m}$ & $69.8(152.) \dagger$ & $6.75(35.1)$ & $0.90(5.85)$ & $\cdots(0.45)$ & $\cdots(\cdots)$ & $\cdots(\cdots)$ & $7.65(41.4) \ddagger$ \\
\hline $\mathrm{H}_{2}(17.03 \mu \mathrm{m})$ & $3.15(10.8) \dagger$ & $\cdots(\cdots)$ & $\cdots(\cdots)$ & $\cdots(\cdots)$ & $\cdots(\cdots)$ & $\cdots(\cdots)$ & $\cdots(\cdots) \ddagger$ \\
\hline [S III] $18.71 \mu \mathrm{m}$ & $10.8(43.2) \dagger$ & $\ldots(3.15)$ & $\cdots(\cdots)$ & $\cdots(\cdots)$ & $\cdots(\cdots)$ & $\cdots(\cdots)$ & $\cdots(3.15) \ddagger$ \\
\hline$[\mathrm{Ne} \mathrm{v}] 24.32 \mu \mathrm{m}$ & $5.85(26.6)$ & $3.15(11.2)$ & $1.80(14.4)$ & $\cdots(0.90)$ & $\cdots(\cdots)$ & $\cdots(\cdots)$ & $10.8(53.1)$ \\
\hline [O IV] $25.89 \mu \mathrm{m}$ & 69.8 (152.) & $20.2(82.3)$ & $27.5(73.8)$ & $0.90(17.1)$ & $\ldots(0.90)$ & $\cdots(\cdots)$ & 118. (326.) \\
\hline [S III] $33.48 \mu \mathrm{m}$ & $26.7(69.8)$ & 35.1 (122.) & $14.4(45.0)$ & $0.90(6.75)$ & $\ldots(1.80)$ & $\cdots(\cdots)$ & 77.1 (245.) \\
\hline$[\mathrm{Si} \mathrm{II}] 34.81 \mu \mathrm{m}$ & 69.8 (210.) & 122. (247.) & 73.8 (174.) & $17.1(54.0)$ & $1.80(28.8)$ & $\cdots(0.90)$ & 284. (715.) \\
\hline [O III] $51.81 \mu \mathrm{m}$ & 210. (464.) & 122. (247.) & 73.8 (246.) & $17.1(54.0)$ & $0.90(1.80)$ & $\cdots(\cdots) \dagger$ & 424. (1013.) $\ddagger$ \\
\hline$[\mathrm{N}$ III $] 57.32 \mu \mathrm{m}$ & 43.2 (106.) & 20.2 (82.3.) & $0.90(14.4)$ & $\cdots(\cdots)$ & $\cdots(\cdots)$ & $\cdots(\cdots)$ & 64.3 (203.) \\
\hline$[\mathrm{OI}] 63.18 \mu \mathrm{m}$ & 366. (687.) & 459. (856.) & 333. (563.) & 54. (138.) & $14.8(47.7)$ & $\cdots(2.25) \dagger$ & 1227. (2294.) $\ddagger$ \\
\hline [O III] $88.35 \mu \mathrm{m}$ & 366. (571.) & 177. (459.) & 117. (246.) & $32.8(88.2) \dagger$ & $1.80(28.8) \dagger$ & $\cdots(0.45) \dagger$ & 695. (1393.) $\ddagger$ \\
\hline
\end{tabular}

Notes. $\uparrow:$ outside the SAFARI spectral range; $\ddagger$ : excluding detections outside the SAFARI spectral range.

$$
\begin{aligned}
& \log \left(L_{\left[\mathrm{N}_{\mathrm{III}}\right] 57.32}\right)=(0.78 \pm 0.10) \log \left(L_{\mathrm{IR}}\right)-(\mathbf{2 . 7 8} \pm \mathbf{0 . 3 5}) \\
& \text { with } R=0.94, n=10, \chi^{2}=0.14 \text {, } \\
& \log \left(L_{[\mathrm{O} \mathrm{I}] 63.18}\right)=(0.98 \pm 0.03) \log \left(L_{\mathrm{IR}}\right)-(\mathbf{2 . 9 5} \pm \mathbf{0 . 1 1}) \\
& \text { with } R=0.94, n=109, \chi^{2}=9.1 \text {, } \\
& \log \left(L_{[\mathrm{O} \text { III }] 88.36}\right)=(0.98 \pm 0.10) \log \left(L_{\mathrm{IR}}\right)-(\mathbf{3 . 1 1} \pm \mathbf{0 . 3 3}) \\
& \text { with } R=0.81, n=55, \chi^{2}=12.7 \text {, } \\
& \log \left(L_{[\mathrm{N} \text { II] } 121.9}\right)=(1.01 \pm 0.04) \log \left(L_{\mathrm{IR}}\right)-(\mathbf{3 . 8 0} \pm \mathbf{0 . 1 2}) \\
& \text { with } R=0.93, n=100, \chi^{2}=13.3 \text {, } \\
& \log \left(L_{[\mathrm{O} \mathrm{I}] 145.5}\right)=(0.89 \pm 0.06) \log \left(L_{\mathrm{IR}}\right)-(\mathbf{3 . 7 8} \pm \mathbf{0 . 1 9}) \\
& \text { with } R=0.91, n=46, \chi^{2}=10.0 \text {, } \\
& \log \left(L_{\left[\mathrm{C}_{\text {II }}\right] 157.7}\right)=(0.89 \pm 0.03) \log \left(L_{\mathrm{IR}}\right)-(\mathbf{2 . 6 7} \pm \mathbf{0 . 0 8}) \\
& \text { with } R=0.92, n=217 \cdot \chi^{2}=42.3 \text {. }
\end{aligned}
$$

For all correlations the hypothesis that the variables are unrelated can be rejected at a level of significance which is always less than $10^{-3}$. Wu et al. (2010) presented the relation between the total infrared luminosity and the PAH emission band at $11.25 \mu \mathrm{m}$ for AGNs and starburst galaxies of the $24 \mu \mathrm{m}$ flux limited intermediate redshift $(\langle z\rangle \sim 0.14)$ sample of 5MUSES (Wu et al. 2011). Their result is comparable to ours. By inverting our relations, we derive a slope of $1.05 \pm 011$ for the Seyfert galaxies and $0.85 \pm 0.08$ for the starburst galaxies, compared to their slopes of $1.00 \pm 0.04$ and $0.98 \pm 0.03$, respectively.

\section{RESULTS}

\subsection{Number Counts per Spectral Line}

To compare and visualize our results for the three galaxy evolution models, we need to adopt a line detection sensitivity curve as a function of wavelength $\lambda$, an integration time, and a field of view (FoV) for the simulated observations. For this purpose, we opt to use numbers relevant to future missions or facilities. For the FIR domain, we use the sensitivity curve proposed for SPICA's far-IR instrument (SAFARI), while for the submillimeter domain, we use the sensitivity curve that has been estimated for an $R=$ 1000 resolution spectrometer at the focal plane of the CCAT telescope. Details on these instruments are presented in the Appendix. Moreover, we selected a common integration time of $1 \mathrm{hr}$ and a total FoV to be covered by our simulated survey of $0.5 \mathrm{deg}^{2}$. For an instrument, such as SAFARI, with a $2^{\prime} \times 2^{\prime}$ FoV, this corresponds to $450 \mathrm{hr}$ of integration time, to be compared to $4.5 \mathrm{hr}$ for a CCAT spectrometer, assuming a FoV of $5^{\prime} \times 5^{\prime}$.

The resulting number of AGN and starbursts that will be detectable in each line as a function of $z$ with SAFARI is presented in Tables 1 and 2, respectively, for the Franceschini et al. (2010) model. The same results are presented in Tables 3 and 4 for the Gruppioni et al. (2011) model. The number of detectable galaxies based on the Valiante et al. (2009) model is presented in Table 5. We note that for the sake of completeness and to assist further planning and designing of new instrumentation, we also present in Tables 1-5 the predicted number of sources that are detectable in the low- $z$ bins for the short-wavelength lines and those in the 
Table 2

Number of Starburst Galaxies Detectable in a SAFARI Survey of $0.5 \mathrm{deg}^{2}$ (450 Pointings with a FoV of $2^{\prime} \times 2^{\prime}$ ) in IR Lines as a Function of Redshift at $5 \sigma(3 \sigma)$ in $1 \mathrm{hr}$ Integration per FoV, Following Franceschini et al. (2010)

\begin{tabular}{|c|c|c|c|c|c|c|c|}
\hline Line/Redshift & $0<z<0.75$ & $0.75<z<1.25$ & $1.25<z<1.75$ & $1.75<z<2.25$ & $2.25<z<2.75$ & $2.75<z<4$ & All Redshifts \\
\hline PAH(11.25 $\mu \mathrm{m})$ & 1257. (1808.) † & 1790. (2330.) $\dagger$ & 984. (1498.) $\dagger$ & 277. (519.) & 105. (242.) & $6.30(19.4)$ & 388. (780.) $\ddagger$ \\
\hline$[\mathrm{Ne}$ II $] 12.81 \mu \mathrm{m}$ & 355. (636.) † & 258. (495.) $\dagger$ & 101. (161.) & $22.9(72.9)$ & $2.25(20.2)$ & $\ldots(0.45)$ & 126. $(255.) \ddagger$ \\
\hline [Ne III] $15.55 \mu \mathrm{m}$ & $43.2(70.6) \dagger$ & $16.6(50.8)$ & $7.65(37.3)$ & $1.80(2.70)$ & $\ldots(0.45)$ & $\cdots(\cdots)$ & $26.0(91.2) \ddagger$ \\
\hline $\mathrm{H}_{2}(17.03 \mu \mathrm{m})$ & $4.95(17.5) \dagger$ & $1.80(4.95)$ & $\cdots(1.35)$ & $\cdots(\cdots)$ & $\cdots(\cdots)$ & $\cdots(\cdots)$ & $1.80(6.0) \ddagger$ \\
\hline$[\mathrm{S}$ III $] 18.71 \mu \mathrm{m}$ & $70.6(176.) \dagger$ & $16.6(50.8)$ & $2.25(19.3)$ & $0.45(1.80)$ & $\cdots(1.35)$ & $\cdots(\cdots)$ & $19.3(73.2) \ddagger$ \\
\hline [O IV] $25.89 \mu \mathrm{m}$ & $\ldots(1.35)$ & $\cdots(0.45)$ & $\ldots(0.45)$ & $\cdots(\cdots)$ & $\cdots(\cdots)$ & $\cdots(\cdots)$ & $\cdots(2.25)$ \\
\hline [S III] $33.48 \mu \mathrm{m}$ & 176. (355.) & 258. (495.) & 161. (337.) & 44.1 (119.) & $20.2(64.3)$ & $0.45(6.30)$ & 660.(1040.) \\
\hline [Si II] $34.81 \mu \mathrm{m}$ & 254. (482.) & 364. (692.) & 239. (460.) & $72.9(187)$. & 39.1 (105.) & $0.90(6.30)$ & 970. (1932.) \\
\hline [O III] $51.81 \mu \mathrm{m}$ & 355. (816.) & 176. (364.) & 101. (337.) & $22.9(72.9)$ & $0.45(2.25)$ & $\cdots(\cdots) \dagger$ & 655. (1592.) $\ddagger$ \\
\hline$[\mathrm{N}$ III $] 57.32 \mu \mathrm{m}$ & 70.6 (176.) & 29.7 (118.) & $1.35(19.3)$ & $\ldots(0.45)$ & $\cdots(\cdots)$ & $\cdots(\cdots)$ & 102. (314.) \\
\hline [O I] $63.18 \mu \mathrm{m}$ & 636. (1257.) & 692. (1340.) & 460. (783.) & 72.9. (187.) & $20.2(64.3)$ & $\cdots(2.70) \dagger$ & 1881. (3634.) $\ddagger$ \\
\hline [O III] $88.35 \mu \mathrm{m}$ & 636. (1023.) & 258. (692.) & 161. (337.) & $44.1(119.) \dagger$ & $2.25(39.1) \dagger$ & $\cdots(0.45) \dagger$ & 1101. (2211.) $\ddagger$ \\
\hline
\end{tabular}

Notes. $\uparrow:$ outside the SAFARI spectral range; $\ddagger$ : excluding detections outside the SAFARI spectral range.

Table 3

Number of AGNs Detectable in a SAFARI Survey of $0.5 \mathrm{deg}^{2}\left(450\right.$ Pointings with a FoV of $\left.2^{\prime} \times 2^{\prime}\right)$ in IR Lines as a Function of Redshift at $5 \sigma(3 \sigma)$ in $1 \mathrm{hr}$ Integration per FoV, Following Gruppioni et al. (2011)

\begin{tabular}{|c|c|c|c|c|c|c|c|}
\hline Line/Redshift & $0<z<0.75$ & $0.75<z<1.25$ & $1.25<z<1.75$ & $1.75<z<2.25$ & $2.25<z<2.75$ & $2.75<z<4$ & All Redshifts \\
\hline PAH(11.25 $\mu \mathrm{m})$ & 814. (1057.) $\dagger$ & 512. $(1076.) \dagger$ & 121. (310.)† & $12.6(64.3)$ & $3.60(13.9)$ & $\cdots(\cdots)$ & $16.2(78.2) \ddagger$ \\
\hline [Ne II] $12.81 \mu \mathrm{m}$ & 240. (398.) $\dagger$ & $16.6(111.) \dagger$ & $1.80(9.90)$ & $0.45(1.80)$ & $\cdots(0.45)$ & $\cdots(\cdots)$ & $2.25(12.1) \ddagger$ \\
\hline$[\mathrm{Ne} \mathrm{v}] 14.32 \mu \mathrm{m}$ & $82.3(177.) \dagger$ & $3.15(9.90) \dagger$ & $\ldots(0.90)$ & $\cdots(\cdots)$ & $\cdots(\cdots)$ & $\cdots(\cdots)$ & $\ldots(0.90) \ddagger$ \\
\hline [Ne III $] 15.55 \mu \mathrm{m}$ & 240. (398.) $\dagger$ & $3.15(16.6)$ & $1.80(5.85)$ & $0.45(1.80)$ & $\cdots(\cdots)$ & $\cdots(\cdots)$ & $5.40(24.2) \ddagger$ \\
\hline $\mathrm{H}_{2}(17.03 \mu \mathrm{m})$ & $16.2(51.3) \dagger$ & $\cdots(0.45)$ & $\cdots(\cdots)$ & $\cdots(\cdots)$ & $\cdots(\cdots)$ & $\cdots(\cdots)$ & $\cdots(0.45) \ddagger$ \\
\hline [S III $] 18.71 \mu \mathrm{m}$ & $82.3(177.) \dagger$ & $\ldots(1.80)$ & $\ldots(0.45)$ & $\cdots(\cdots)$ & $\cdots(\cdots)$ & $\cdots(\cdots)$ & $\cdots(2.25) \ddagger$ \\
\hline$[\mathrm{Ne} \mathrm{v}] 24.32 \mu \mathrm{m}$ & 51.3 (124.) & $1.80(5.40)$ & $3.15(9.90)$ & $0.45(3.60)$ & $\cdots(0.45)$ & $\cdots(\cdots)$ & 56.7 (143.) \\
\hline [O IV] $25.89 \mu \mathrm{m}$ & 240. (491.) & $9.90(58.0)$ & $17.6(47.7)$ & $3.60(22.0)$ & $0.90(3.60)$ & $\cdots(\cdots)$ & 272. (622.) \\
\hline [S III] $33.48 \mu \mathrm{m}$ & 124. (240.) & $16.6(111)$. & $9.90(28.8)$ & $3.60(12.6)$ & $0.45(6.75)$ & $\cdots(\cdots)$ & 155. (399.) \\
\hline$[\mathrm{Si} \mathrm{II}] 34.81 \mu \mathrm{m}$ & 240.(398.) & 111. (326.) & 47.7 (121.) & $22.0(64.3)$ & $6.75(45.9)$ & $\cdots(0.45)$ & 427. (956.) \\
\hline [O III $] 51.81 \mu \mathrm{m}$ & 592. (814.) & 111. (326.) & 47.7 (188.) & $22.0(64.3)$ & $3.60(6.75)$ & $\cdots(\cdots)$ & 776. (1399.) \\
\hline$[\mathrm{N} \mathrm{III]} 57.32 \mu \mathrm{m}$ & 177. (398.) & $9.90(58.0)$ & $1.80(9.90)$ & $\ldots(0.90)$ & $\cdots(\cdots)$ & $\cdots(\cdots)$ & 189. (467.) \\
\hline [O I] $63.18 \mu \mathrm{m}$ & 701. (1057.) & 762. (1442.) & 310. (759.) & 64.3 (148.) & $25.2(75.6)$ & $\cdots(1.80) \dagger$ & 1864. (3483.) $\ddagger$ \\
\hline [O III $] 88.35 \mu \mathrm{m}$ & 701. (934.) & 192. (762.) & 74.2 (188.) & $39.1(100.) \dagger$ & $6.75(45.9) \dagger$ & $\cdots(\cdots) \dagger$ & 967. (1884.) $\ddagger$ \\
\hline
\end{tabular}

Notes. $\uparrow:$ outside the SAFARI spectral range; $\ddagger$ : excluding detections outside the SAFARI spectral range.

Table 4

Number of Starburst Galaxies Detectable in a SAFARI Survey of $0.5 \mathrm{deg}^{2}$ (450 Pointings with a FoV of $2^{\prime} \times 2^{\prime}$ ) in IR Lines as a Function of Redshift at $5 \sigma(3 \sigma)$ in $1 \mathrm{hr}$ Integration per FoV, Following Gruppioni et al. (2011)

\begin{tabular}{|c|c|c|c|c|c|c|c|}
\hline Line/Redshift & $0<z<0.75$ & $0.75<z<1.25$ & $1.25<z<1.75$ & $1.75<z<2.25$ & $2.25<z<2.75$ & $2.75<z<4$ & All Redshifts \\
\hline PAH(11.25 $\mu \mathrm{m})$ & 515. (779.)† & 329. (387.)† & 373. (469.) $\dagger$ & 140. (240.) & $48.1(99.0)$ & $4.05(13.9)$ & 192. (353.) $\ddagger$ \\
\hline [Ne II] $12.81 \mu \mathrm{m}$ & 153. (256.) $\dagger$ & 122. (180.)† & $64.8(92.7)$ & $20.7(48.1)$ & 4.05 (11.7) & $\cdots(0.45)$ & $89.6(153.) \ddagger$ \\
\hline [Ne III] $15.55 \mu \mathrm{m}$ & $34.6(62.1) \dagger$ & $15.7(36.4)$ & $11.2(29.2)$ & $4.05(7.65)$ & $0.45(0.90)$ & $\cdots(\cdots)$ & $31.4(74.1) \ddagger$ \\
\hline $\mathrm{H}_{2}(17.03 \mu \mathrm{m})$ & $15.3(20.2) \dagger$ & $3.60(5.85)$ & $0.45(3.15)$ & $\cdots(0.45)$ & $\cdots(\cdots)$ & $\cdots(\cdots)$ & $4.05(9.45) \ddagger$ \\
\hline [S III] $18.71 \mu \mathrm{m}$ & $62.1(115.) \dagger$ & $15.7(36.5)$ & $6.30(18.5)$ & $0.90(4.05)$ & $0.45(2.20)$ & $\cdots(\cdots)$ & $23.3(61.2) \ddagger$ \\
\hline [O IV] $25.89 \mu \mathrm{m}$ & $3.60(8.10)$ & $0.45(1.80)$ & $0.45(1.35)$ & $\ldots(0.45)$ & $\cdots(\cdots)$ & $\cdots(\cdots)$ & $4.50(10.7)$ \\
\hline [S III] $33.48 \mu \mathrm{m}$ & 85.0 (153.) & 122. (180.) & 92.7 (171.) & $32.4(71.1)$ & $11.7(31.5)$ & $0.45(2.25)$ & 344. (609.) \\
\hline [Si II] $34.81 \mu \mathrm{m}$ & 153. (256.) & 150. (212.) & 128. (219.) & 48.1 (102.) & $19.8(48.1)$ & $0.90(4.05)$ & 500. (841.) \\
\hline$[\mathrm{O}$ III $] 51.81 \mu \mathrm{m}$ & 256. (412.) & 95.4 (150.) & 64.8 (171.) & $20.7(48.1)$ & $0.90(4.00)$ & $\cdots(\cdots)$ & 438. (785.) \\
\hline$[\mathrm{N}$ III $] 57.32 \mu \mathrm{m}$ & $62.1(153)$. & 24.7(72.) & $3.15(18.4)$ & $\cdots(0.90)$ & $\cdots(\cdots)$ & $\cdots(\cdots)$ & $90.0(244)$. \\
\hline [O I] $63.18 \mu \mathrm{m}$ & 326. (637.) & 212. (284.) & 219. (321.) & 48.1 (102.) & $11.7(31.5)$ & $\cdots(2.25)$ & 817. (1378.) \\
\hline [O III $] 88.35 \mu \mathrm{m}$ & 326. (515.) & 122. (212.) & 92.7 (171.) & $32.4(71.1) \dagger$ & $4.05(19.8) \dagger$ & $\ldots(0.45) \dagger$ & 244. (898.) \\
\hline
\end{tabular}

Notes. $\nmid$ : outside the SAFARI spectral range; $\ddagger$ : excluding detections outside the SAFARI spectral range.

high- $z$ bins for the long-wavelength lines, even lines outside the nominal SAFARI spectral range. We have simply assumed a flat extrapolation of the SAFARI sensitivities to shorter and longer wavelengths. Tables 6-8 present the simulation results for CCAT.

A basic result of this analysis is that the total number of detectable objects agrees to within a factor of two to three for most lines and $z$ ranges, and that at least a thousand galaxies will be simultaneously detected in four lines at $5 \sigma$ over a half-square-degree. A 


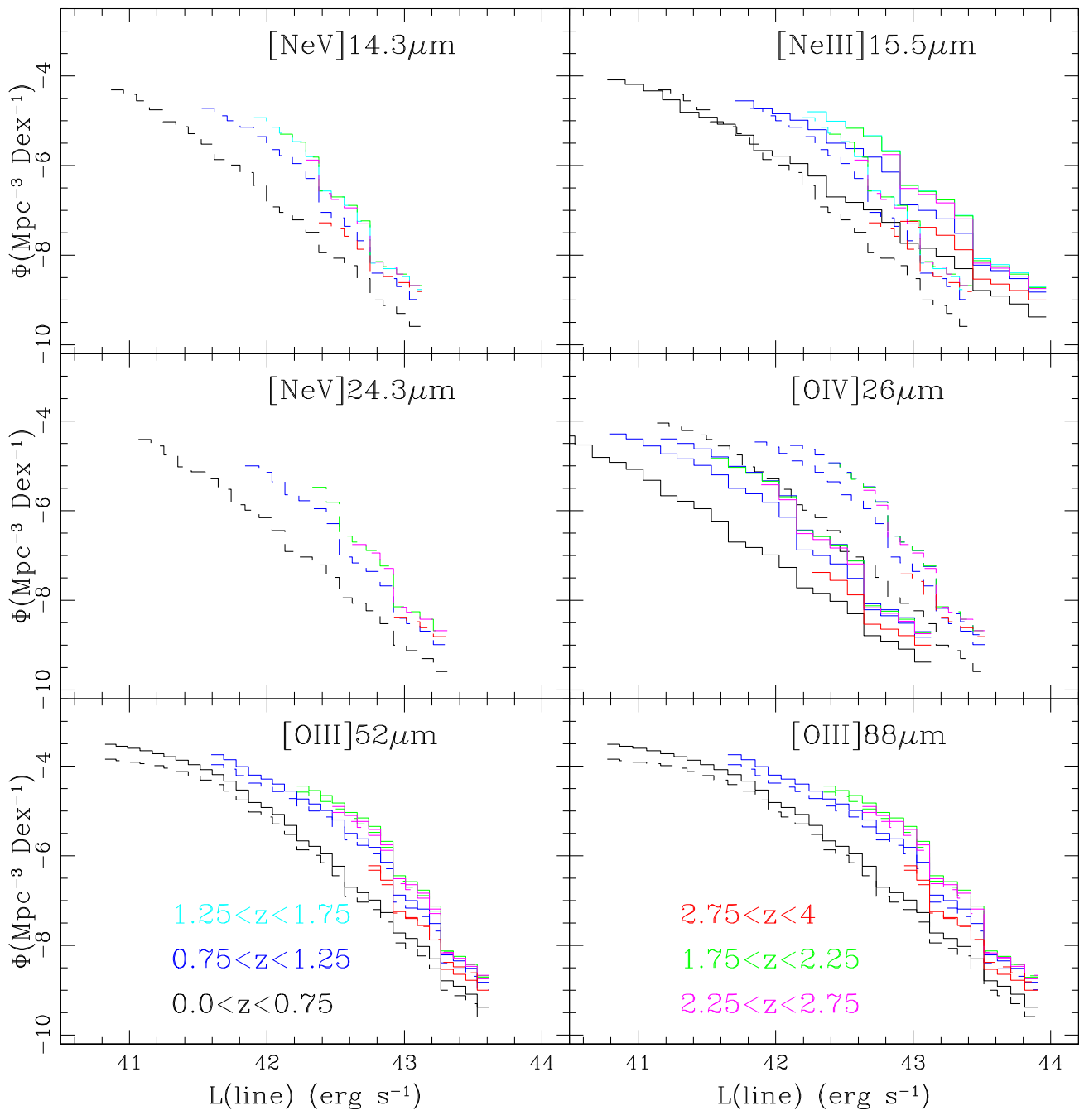

Figure 5. Predicted line luminosity functions of [Ne III] $15.5 \mu \mathrm{m}$, [Ne v] $24.3 \mu \mathrm{m}$, [O IV] $26 \mu \mathrm{m}$, [O III] $52 \mu \mathrm{m}$, and [O III] $88 \mu \mathrm{m}$, for SAFARI. Dashed lines correspond to the predictions for AGN, while solid lines correspond to the predictions for starbursts.

(A color version of this figure is available in the online journal.)

Table 5

Total Number of Galaxies Detectable in a SAFARI Survey of $0.5 \mathrm{deg}^{2}$ in IR Lines as a Function of Redshift at $5 \sigma(3 \sigma)$ in $1 \mathrm{hr}$ Integration per FoV, Following Valiante et al. (2009)

\begin{tabular}{|c|c|c|c|c|c|c|c|}
\hline Line/Redshift & $0<z<0.75$ & $0.75<z<1.25$ & $1.25<z<1.75$ & $1.75<z<2.25$ & $2.25<z<2.75$ & $2.75<z<4$ & All Redshifts \\
\hline $\mathrm{PAH}(11.25 \mu \mathrm{m})$ & $2257(3353) \dagger$ & $879(2383) \dagger$ & $505(1074) \dagger$ & $85.0(322)$ & $29.2(91.3)$ & $\cdots(4.95)$ & $114(418) \ddagger$ \\
\hline$[\mathrm{Ne}$ II $] 12.81 \mu \mathrm{m}$ & $471(850) \dagger$ & $94.0(251) \dagger$ & $7.65(28.8)$ & $\cdots(11.7)$ & $\cdots(\cdots)$ & $\cdots(\cdots)$ & $7.65(40.5) \ddagger$ \\
\hline [Ne III] $15.55 \mu \mathrm{m}$ & $331(638) \dagger$ & $31.0(94.0)$ & $15.7(51.7)$ & $3.60(26.1)$ & $\cdots(7.20)$ & $\cdots(\cdots)$ & $50.3(179) \ddagger$ \\
\hline [S III] $18.71 \mu \mathrm{m}$ & $40.0(103) \dagger$ & $\cdots(2.25)$ & $\cdots(\cdots)$ & $\cdots(\cdots)$ & $\ldots(1.80)$ & $\cdots(\cdots)$ & $\cdots(2.25) \ddagger$ \\
\hline [O IV $] 25.89 \mu \mathrm{m}$ & $331(638)$ & $31.0(94.0)$ & $28.8(83.2)$ & $11.7(49.0)$ & $\cdots(15.7)$ & $\cdots(\cdots)$ & $402(880)$ \\
\hline [S III] $33.48 \mu \mathrm{m}$ & $331(638)$ & $94.0(251)$ & $28.8(83.2)$ & $11.7(49.0)$ & $7.20(29.2)$ & $\cdots(\cdots)$ & $473(1050)$ \\
\hline$[\mathrm{N}$ III $] 57.32 \mu \mathrm{m}$ & $331(850)$ & $31.0(155)$ & $3.60(28.8)$ & $\cdots(\cdots)$ & $\cdots(\cdots)$ & $\cdots(\cdots)$ & 366 (1034) \\
\hline [OI] $63.18 \mu \mathrm{m}$ & $1811(3353)$ & $1263(2383)$ & 745 (1512) & $211(473)$ & 91.3 (207) & $1.80(19.8) \dagger$ & $4121(7928) \ddagger$ \\
\hline [O $\mathrm{III}] 88.35 \mu \mathrm{m}$ & 1811 (2773) & 392. (1263) & $212(505)$ & $136(321) \dagger$ & $29.2(142) \dagger$ & $\ldots(4.95) \dagger$ & $2415(4541) \ddagger$ \\
\hline
\end{tabular}

Notes. $\nmid$ : outside the SAFARI spectral range; $\ddagger$ : excluding detections outside the SAFARI spectral range.

comparison of the output of the three models is plotted in Figure 4 for SAFARI. A survey of the assumed sensitivity will detect bright lines (e.g., [O I $]$ and $[\mathrm{O} \mathrm{III}]$ ) and PAH features in thousands of galaxies at $z>1$. Hundreds of $z>1$ AGNs will be detected in the [O IV] line, and several tens of $z>1$ sources will be detected in [Ne v] and $\mathrm{H}_{2}$. For $\mathrm{H}_{2}$ in particular, this number corresponds to a lower limit. Our models do not account for an increase of the $\mathrm{H}_{2}$ emission efficiency as a cooling mechanism, or for the $\mathrm{H}_{2}$ mass content to increase with increasing $z$ and decreasing metallicity. Neither do the applied continuum-to-line luminosity relations include 


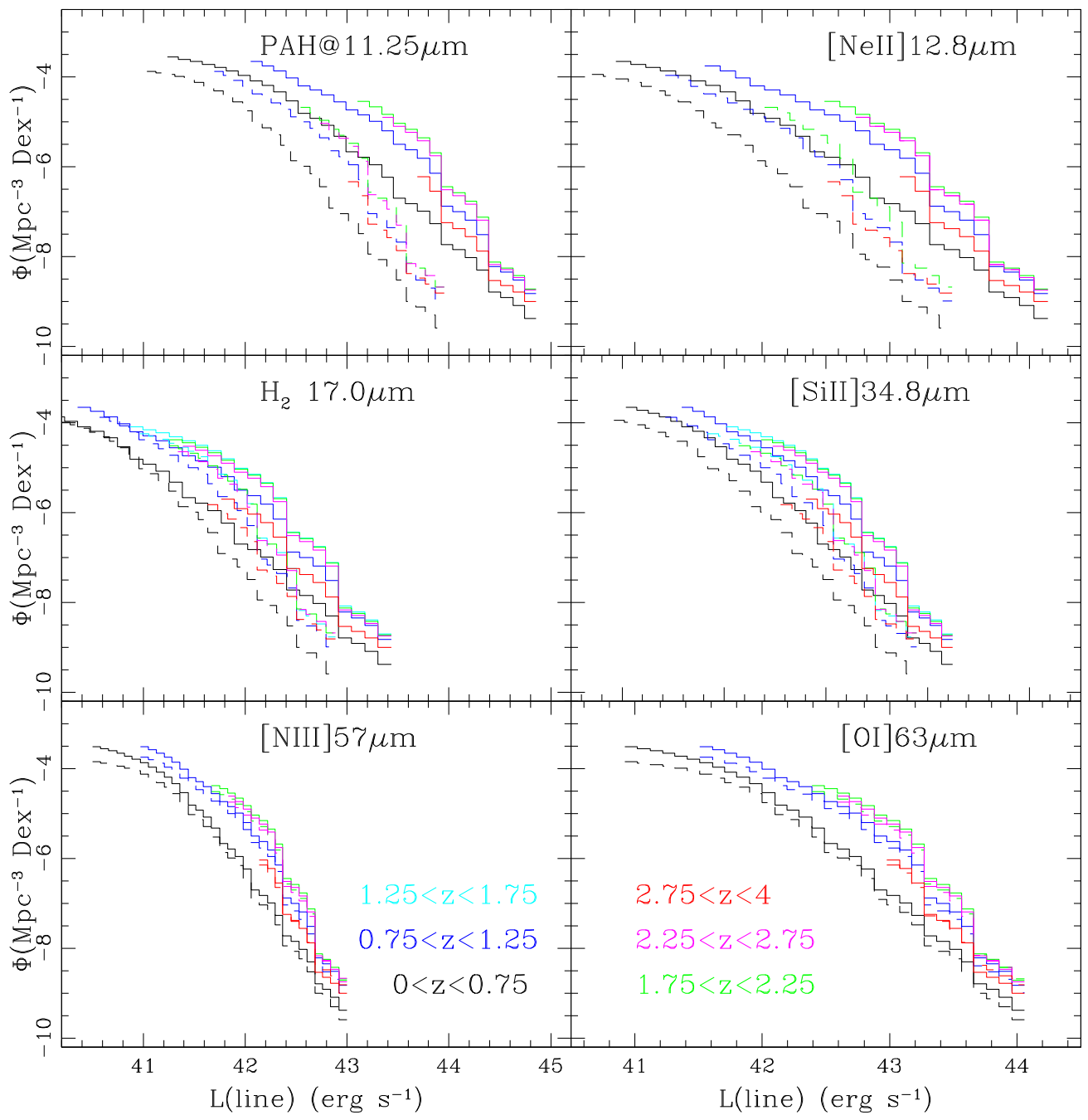

Figure 6. Predicted line luminosity functions of PAH $11.25 \mu \mathrm{m}$, [Ne II] $12.8 \mu \mathrm{m}, \mathrm{H}_{2} 17 \mu \mathrm{m}$, [Si II] $34.8 \mu \mathrm{m}$, and [O I] $63 \mu \mathrm{m}$, for SAFARI. Dashed lines correspond to the predictions for AGN, while solid lines correspond to the predictions for starbursts.

(A color version of this figure is available in the online journal.)

Table 6

Number of Galaxies Detectable in a CCAT Survey of $0.5 \mathrm{deg}^{2}\left(72\right.$ Pointings with a FoV of $\left.5^{\prime} \times 5^{\prime}\right)$ in IR Lines as a Function of Redshift at $5 \sigma(3 \sigma)$ in $1 \mathrm{hr}$ Integration per FoV, Following Franceschini et al. (2010)

\begin{tabular}{|c|c|c|c|c|c|c|}
\hline Line/Redshift & $0<z<0.75$ & $0.75<z<1.25$ & $1.25<z<1.75$ & $1.75<z<2.25$ & $2.25<z<2.75$ & $2.75<z<4$ \\
\hline [O I] $63.18 \mu \mathrm{m}$ & $\ldots(\cdots)$ & $\cdots(\cdots)$ & $\ldots(\cdots)$ & $\cdots(0.29) \diamond$ & $\cdots(\cdots)$ & $\cdots(\cdots)$ \\
\hline [O III] $88.35 \mu \mathrm{m}$ & $\cdots(\cdots)$ & $2.81(13.5) \diamond$ & $0.29(2.80) \dagger$ & ... (2.09) $\ddagger$ & $27.0(85.8) \#$ & $2.59(21.2) \$$ \\
\hline [N II] $121.90 \mu \mathrm{m}$ & $2.74(7.49) \dagger$ & $\cdots(0.22) \dagger$ & $\cdots(\cdots) \ddagger$ & 1.10 (12.2) \# & $0.94(3.17) \$$ & $\ldots(0.22) \S$ \\
\hline [O I] $145.52 \mu \mathrm{m}$ & $\cdots(0.43) \dagger$ & $\cdots(\cdots) \ddagger$ & $\ldots(0.86) \#$ & $\cdots(\cdots) \$$ & $\cdots(\cdots) \S$ & $\cdots(\cdots)$ \\
\hline [C II $] 157.74 \mu \mathrm{m}$ & $95.0(235.) \dagger$ & 922. (1786.) \# & 613. (1312.) \$ & 250. (517.) \$ & $85.8(219.) \S$ & 109. (350.) ब \\
\hline
\end{tabular}

Notes. $\diamond$ : at $200 \mu \mathrm{m}$ band; $\dagger:$ at $230 \mu \mathrm{m}$ band; $\ddagger$ : at $291 \mu \mathrm{m}$ band; \#: at $350 \mu \mathrm{m}$ band; $\$$ : at $450 \mu \mathrm{m}$ band; $\S:$ at $620 \mu \mathrm{m}$ band; $\uparrow:$ at $740 \mu \mathrm{m}$ band.

Table 7

Number of Galaxies Detectable in a CCAT Survey of $0.5 \operatorname{deg}^{2}\left(72\right.$ Pointings with a FoV of $\left.5^{\prime} \times 5^{\prime}\right)$ in IR Lines as a Function of Redshift at $5 \sigma(3 \sigma)$ in $1 \mathrm{hr}$ Integration per FoV, Following Gruppioni et al. (2011)

\begin{tabular}{|c|c|c|c|c|c|c|}
\hline Line/Redshift & $0<z<0.75$ & $0.75<z<1.25$ & $1.25<z<1.75$ & $1.75<z<2.25$ & $2.25<z<2.75$ & $2.75<z<4$ \\
\hline [OI] $63.18 \mu \mathrm{m}$ & $\cdots(\cdots)$ & $\cdots(\cdots)$ & $\cdots(\cdots)$ & $0.50(2.02) \diamond$ & $0.07(0.36) \dagger$ & $\cdots(0.22) \ddagger$ \\
\hline [O III] $88.35 \mu \mathrm{m}$ & $\cdots(\cdots)$ & $5.26(14.0) \diamond$ & $1.51(5.47) \dagger$ & $1.01(7.49) \ddagger$ & 37.9 (108)\# & $11.0(39.7)$ \\
\hline [N II] $121.90 \mu \mathrm{m}$ & $3.17(7.85) \dagger$ & $0.22(0.79) \dagger$ & $0.14(0.72) \ddagger$ & 3.60 (25.3)\# & $2.74(11.0) \S$ & $0.50(1.73) \S$ \\
\hline [C II] $157.74 \mu \mathrm{m}$ & $79.3(172) \dagger$ & 975 (1727)\# & $528(1498)$ & $249(472)$ & $108(240) \S$ & 188 (540) ๆ \\
\hline
\end{tabular}

Notes. $\diamond$ : at $200 \mu \mathrm{m}$ band; $\dagger:$ at $230 \mu \mathrm{m}$ band; $\ddagger$ : at $291 \mu \mathrm{m}$ band; \#: at $350 \mu \mathrm{m}$ band; $\$$ : at $450 \mu \mathrm{m}$ band; $\S:$ at $620 \mu \mathrm{m}$ band; $\uparrow:$ at $740 \mu \mathrm{m}$ band. 
Table 8

Number of Galaxies Detectable in a CCAT Survey of $0.5 \mathrm{deg}^{2}\left(72\right.$ Pointings with a FoV of $\left.5^{\prime} \times 5^{\prime}\right)$ in IR Lines as a Function of Redshift at $5 \sigma(3 \sigma)$ in $1 \mathrm{hr}$ Integration per FoV, Following Valiante et al. (2009)

\begin{tabular}{|c|c|c|c|c|c|c|}
\hline Line/Redshift & $0<z<0.75$ & $0.75<z<1.25$ & $1.25<z<1.75$ & $1.75<z<2.25$ & $2.25<z<2.75$ & $2.75<z<4$ \\
\hline [O I] $63.18 \mu \mathrm{m}$ & $\cdots(\cdots)$ & $\cdots(\cdots)$ & $\cdots(\cdots)$ & $\cdots(\cdots)$ & $\cdots(\cdots)$ & $\cdots(\cdots)$ \\
\hline [O III $] 88.35 \mu \mathrm{m}$ & $\cdots(\cdots)$ & $2.52(9.22) \diamond$ & $0.14(3.67) \dagger$ & $\cdots(12.0) \ddagger$ & 91.4 (208.) \# & $9.94(36.1)$ \\
\hline [N II] $121.90 \mu \mathrm{m}$ & $2.38(7.34) \dagger$ & $\cdots(0.14) \dagger$ & $\cdots(\cdots)$ & 3.74 (49.3) \# & $7.20(29.2)$ & $\ldots(0.07) \S$ \\
\hline [O I] $145.52 \mu \mathrm{m}$ & $0.50(0.94) \dagger$ & $\cdots(\cdots)$ & $\ldots(1.37) \#$ & $\cdots(\cdots)$ & $\cdots(\cdots)$ & $\cdots(\cdots)$ \\
\hline [C II $] 157.74 \mu \mathrm{m}$ & 104. (231.) $\dagger$ & 1264. (2385.) \# & 745. (2059.) & 474. (956.) & 208. (452.) § & 157. (531.) ब \\
\hline
\end{tabular}

Notes. $\diamond:$ at $200 \mu \mathrm{m}$ band; $\dagger:$ at $230 \mu \mathrm{m}$ band; $\ddagger$ : at $291 \mu \mathrm{m}$ band; \#: at $350 \mu \mathrm{m}$ band; $\$$ : at $450 \mu \mathrm{m}$ band; $\S:$ at $620 \mu \mathrm{m}$ band; $9:$ at $740 \mu \mathrm{m}$ band.

sources of extremely high $L\left(\mathrm{H}_{2}\right) / L_{\mathrm{IR}}$ ratios, associated with shock fronts due to galaxy collisions or AGN feedback mechanisms (Cluver et al. 2010; Ogle et al. 2010).

\section{REFERENCES}

Altieri, B., Berta, S., Lutz, D., et al. 2010, A\&A, 518, L17

Bernard-Salas, J., Spoon, H. W. W., Charmandaris, V., et al. 2009, ApJS, 184, 230

Berta, S., Magnelli, B., Lutz, D., et al. 2010, A\&A, 518, L30

Brauher, J. R., Dale, D. A., \& Helou, G. 2008, ApJS, 178, 280

Clegg, P. E., Ade, P. A. R., Armand, C., et al. 1996, A\&A, 315, L38

Cluver, M. E., Appleton, P. N., Boulanger, F., et al. 2010, ApJ, 710, 248

Dale, D. A., Smith, J. D. T., Armus, L., et al. 2006, ApJ, 646, 161

Franceschini, A., Rodighiero, G., Vaccari, M., et al. 2010, A\&A, 517, 74

Genzel, R., Lutz, D., Sturm, E., et al. 1998, ApJ, 498, 579

Glenn, J., Conley, A., Béthermin, M., et al. 2010, MNRAS, 409, 109

Gruppioni, C., Pozzi, F., Zamorani, G., \& Vignali, C. 2011, MNRAS, 416, 70

Houck, J. R., Roellig, T. L., van Cleve, J., et al. 2004, ApJS, 154, 18

Kessler, M. F., Steinz, J. A., Anderegg, M. E., et al. 1996, A\&A, 315, L27

Ogle, P., Boulanger, F., Guillard, P., et al. 2010, ApJ, 724, 1193

Oliver, S., Wang, L., Smith, A. J., et al. 2010, A\&A, 518, L21

Sanders, D. B., \& Mirabel, I. F. 1996, ARA\&A, 34, 749

Smith, J. D. T., Draine, B. T., Dale, D. A., et al. 2007, ApJ, 656, 770

Spinoglio, L., \& Malkan, M. A. 1992, ApJ, 399, 504

Tommasin, S., Spinoglio, L., Malkan, M., \& Fazio, G. 2010, ApJ, 709, 1257

Tommasin, S., Spinoglio, L., Malkan, M. A., et al. 2008, ApJ, 676, 836

Valiante, E., Lutz, D., Sturm, E., Genzel, R., \& Chapin, E. L. 2009, ApJ, 701, 1814

Werner, M. W., Roellig, T. L., Low, F. J., et al. 2004, ApJS, 154, 1

Wu, Y., Helou, G., Armus, L., et al. 2010, ApJ, 723, 895

Wu, Y., Shi, Y., Helou, G., et al. 2011, ApJ, 734, 40 